\title{
SYNANTHROPIC ORCHARD FLORA IN WEST MAZOVIA - CENTRAL POLAND
}

\author{
Jerzy Lisek \\ Research Institute of Horticulture, Konstytucji 3 Maja 1/3 \\ 96-100 Skierniewice, POLAND \\ e-mail: Jerzy.Lisek@inhort.pl
}

(Received September 11, 2012/Accepted November 5, 2012)

A B S T R A C T

Research on synanthropic flora was conducted in the orchards of central Poland (near Skierniewice, Łowicz and Grójec). In the 2007-2010 time period, 390 phytosociological releves were taken, which included herbicide fallow under trees, swarm of inter-rows, tillage places, trodden and rutty places, roadsides, boundary stripes and drainage ditches. In the examined orchards the occurrence of 186 species belonging to 39 botanical families was noted. The most numerously represented were: Asteraceae (21\%), Poaceae (15\%), Brassicacea (8\%) and Fabaceae (7\%). In the examined orchards, $60 \%$ of the found species occurred occasionally or rarely. Those species which were found at the $15 \%$ level were: Equisetum arvense, Polygonum aviculare, Chenopodium album, Amaranthus retroflexus, Stellaria media, Capsella bursa-pastoris, Convolvulus arvensis, Viola arvensis, Epilobium adenocaulon, Conyza canadensis, Taraxacum officinale, Poa annua, and Echinochloa crus-galli. All of these species which occurred at the $15 \%$ level were most frequently noted in the herbicide fallow and were recognised as the most significant orchard weeds. The most numerous group in the examined orchards was made up of the therophytes (50\%), which predominated over hemicryptophytes (31\%) and geophytes $(10 \%)$. Apophytes - native species (59\%) predominated over archeophytes (33\%) and kenophytes $(8 \%)$. Within the vascular flora of the examined orchards, those which were predominant were the segetal species $(26 \%)$, ruderal species $(21 \%)$ and meadow species (19\%).

Key words: Orchard, synanthropic plants, weeds 


\section{INTRODUCTION}

The work aimed to identify the most significant and expansive weeds as well as to assess the diversity of synanthropic flora of commercial orchards in the central part of Poland, taking human activity into account. Results of floristic research enable better identification of risks caused by weeds as well as proper modification of weed control programmes. In Poland, there are few publications concerning those issues. The most inclusive description of the synanthropic flora of commercial orchards is presented by Wróbel (1999). Change dynamics in weed infestation influenced by the use of herbicides over several years, are described by Lipecki (2004). Weed infestation in an organic orchard, where the soil was mulched with compost and treated mechanically, was the subject of research conducted in central Poland (Mika, 2004). In an orchard with Integrated Pest Management (IPM), weed infestation, including species composition, is dependent on the method of weed control and meteorological factors (Markuszewski and Kopytowski, 2008).

Authors evaluating the species composition of orchard flora, both in Poland and abroad, pay special attention to the fundamental role played by human activity, including the use of herbicides and other means of protection against weeds (Conticello and Gandullo, 1991; Jung et al., 1997; Novo et al., 2000; Lisek, 2001; Harrington et. al., 2002; Rifai et al., 2002; Mashaly and Awad, 2003;
Tasseva, 2005; Ustuner and Ustuner, 2011). Occurrence and number of plants constituting synanthropic flora, together with weeds - most important from the economical point of view depend to a substantial degree, on their biology and environmental conditions (Tymrakiewicz, 1976; Mowszowicz, 1984).

\section{MATERIAL AND METHODS}

Field examinations were conducted in the West Mazovian Region of central Poland near Skierniewice (Skierniewice - Pomological Orchard, Dąbrowice, Julków, Strobów), Łowicz (Ostrowiec, Maurzyce) and Grójec (Belsk Duży, Lewiczyn, Błędów, Wólka Łęczeszycka, Goliany). The Braun-Blanquet method was used to analyse 390 phytosociological releves, taken in the years 2007-2010. The orchards were most often located on a flat area and podsolic soil graded Class IV, rarely III or V. The soil was slightly acidic ( $\mathrm{pH}$ 6.0-6.7). Soil from three selected sites (Dąbrowice, Julków, Ostrowiec) was characterised by the following mechanical composition: sand: $69-84 \%$, silt: $12-30 \%$, clay: $1-4 \%$, and soil which contained $1.5-1.6 \%$ of organic matter. The research was conducted in large commercial orchards belonging to 40 specialised horticultural farms cultivating apples, pears, plums, sour cherries, sweet cherries, and peaches. The research was also conducted in 2 experimental orchards. The soil of the newly established orchards was cultivated mechanically, with limited use of herbicides in the row of trees. Starting with the second 
or third year the inter-rows were grassed with long-lasting and frequently mowed meadow grasses. In the tree rows, post-emergence herbicides were applied 2-4 times a year (glyphosate, MCPA and glufosinate were used most often). The following features characterised $80 \%$ of the assessed orchard: 3-15 years old, herbicide fallow under trees, and grassed interrows. Species composition of flora was evaluated from April to September. Names of taxa were given after Mirek et al. (2002) as amended. Taxa were analysed according to the following criteria:

Frequency of occurrence in the investigated area:

1 - occasionally ( $\leq 5 \%$ of sites); 2 - rarely (5.1-10\% of sites); 3 average (10.1-30\% of sites); 4 often (30.1 - 50\% of sites); 5 - very often (> $50.1 \%$ of sites).

Life form according to Raunkiaer (Rutkowski, 2007):

- perennials: $\mathrm{M}$ - megaphanerophyte - tree; N - nanophanerophyte shrub; Ch - herbaceous chamaephyte; $\mathrm{G}$ - geophyte; $\mathrm{H}$ hemicryptophyte;

- short-lived: T1 - annual therophyte; $\mathrm{T} 2$ - biennial therophyte.

Geographical-historical classification according to Kornaś (1981), Zając and Zając (1992):

- Ap. - apophyte; Arch. archeophyte; Ken. - kenophyte; Erg. - ergasiophyte.

Socio-ecological groups according Ellenberg et al. (1992):
- DW - deciduous woodland species; CD - coniferous woodland species; SE - shrub edges species; M meadow species; SS - sandy sward species; XS - xerothermic sward species; RD - ruderal species; SG segetal species; SW - swamp species; N - species of under classification.

Sites of occurrence in investigated orchards:

- TP - tillage places; HF herbicide fallow under trees; SI swarm of inter-rows; TR trodden and rutty places; RS roadsides: BS - boundary stripes; $\mathrm{D}$ - drainage ditches.

\section{RESULTS}

Frequency of occurrence of vascular plants. From among 186 vascular plants, 112 species $(60 \%)$ occurred occasionally or rarely, including Consolida regalis, Malva sylvestris, Lythrum salicilaria, Anagalis arvensis, Stachys palustris (Tab. 1). Plants of 27 species (15\% of the total number), eg. Equisetum arvense, Polygonum aviculare, Chenopodium album, Amaranthus retroflexus, Stellaria media, Capsella bursa-pastoris, Convolvulus arvensis, Viola arvensis, Epilobium adenocaulon, Conyza canadensis, Taraxacum officinale, Роа аппиа, Echinochloa crus-galli, occurred frequently of very frequently.

Species affiliation with botanical families. Plants growing in the 


\section{J. Lisek}

Table 1. Synanthropic flora of commercial orchards in West Mazovia - central Poland, 2007-2010

\begin{tabular}{|c|c|c|c|c|c|}
\hline Families and species & 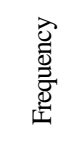 & $\begin{array}{l}\Xi \\
\stackrel{0}{0} \\
\stackrel{0}{3}\end{array}$ & 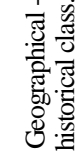 & 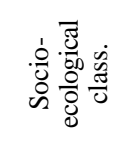 & $\stackrel{0}{=0}$ \\
\hline $\begin{array}{c}\text { Equisetaceae } \\
\text { Equisetum arvense } \mathrm{L} .\end{array}$ & 4 & $G$ & $A \mathrm{p}$ & SG & HF BS \\
\hline Equisetum arvense L. & 4 & U & Ap. & $S U$ & HF, BS \\
\hline $\begin{array}{l}\text { Juglandaceae } \\
\text { Juglans regia L. }\end{array}$ & 1 & M & Erg. & & HF, BS \\
\hline Cannabaceae & & & & & \\
\hline Humulus lupulus $\mathrm{L}$. & 1 & $\mathrm{H}$ & Ap. & DW, SE & $\mathrm{BS}, \mathrm{HF}$ \\
\hline Urticaceae & & & & & \\
\hline Urtica dioica $\mathrm{L}$. & 3 & $\mathrm{H}$ & Ap. & $\mathrm{RD}, \mathrm{SE}$ & HF, BS \\
\hline Urtica urens $\mathrm{L}$. & 3 & $\mathrm{~T} 1$ & Arch. & SG & $\mathrm{HF}, \mathrm{TP}$ \\
\hline $\begin{array}{l}\text { Polygonaceae } \\
\text { Polyoonum amnhibium } \mathrm{yar} \text { terrestre }\end{array}$ & & & & & \\
\hline Polygonum amphibium L. var. terrestre & 1 & $\mathrm{G}$ & Ap. & SW & BS, HF \\
\hline Polygonum aviculare $\mathrm{L}$. & 4 & $\mathrm{~T} 1$ & Ap. & $\mathrm{RD}$ & HF, TP, RS, TR \\
\hline Polygonum persicaria L. & 3 & T1 & Ap. & SG & TP, HF, BS \\
\hline $\begin{array}{l}\text { Polygonum lapathifolium } \quad \text { L. } \\
\text { lapathifolium }\end{array}$ & 1 & $\mathrm{~T} 1$ & Ap. & SW & BS, D \\
\hline Fallopia convolvulus (L.) A. Löve & 3 & $\mathrm{~T} 1$ & Arch. & & $\mathrm{HF}, \mathrm{TP}$ \\
\hline Rumex acetosella $\mathrm{L}$. & 3 & $\mathrm{G}$ & Ap. & $\mathrm{SS}, \mathrm{CW}$ & HF, SI, BS \\
\hline Rumex acetosa $\mathrm{L}$. & 2 & $\mathrm{H}$ & Ap. & & BS, SI, RS, HF \\
\hline Rumex crispus $\mathrm{L}$. & 2 & $\mathrm{H}$ & Ap. & $\mathrm{RD}$ & SI, HF, BS, RS \\
\hline Rumex obtusifolius $\mathrm{L}$. & 1 & $\mathrm{H}$ & Ap. & $\mathrm{RD}$ & SI, HF, BS \\
\hline Chenopodiaceae & & & & & \\
\hline Chenopodium album $\mathrm{L}$. & 4 & $\mathrm{~T} 1$ & Ap. & SG & HF, TP \\
\hline Chenopodium polyspermum $\mathrm{L}$. & 1 & $\mathrm{~T} 1$ & Ap. & SG & TP, HF \\
\hline Atriplex patula $\mathrm{L}$. & 2 & $\mathrm{~T} 1$ & Ap. & SG & HF, TP \\
\hline $\begin{array}{l}\text { Amaranthaceae } \\
\text { Amaranthus retroflexus } \mathrm{L} \text {. }\end{array}$ & 4 & $\mathrm{~T} 1$ & Ken. & SG & HF, TP \\
\hline Caryophyllaceae & & & & & \\
\hline Arenaria serpyllifolia $\mathrm{L}$. & 3 & $\mathrm{~T} 1$ & Ap. & SS & HF, SI, RS \\
\hline Stellaria media (L.) Vill. & 5 & $\mathrm{~T} 1$ & Ap. & SG & $\mathrm{HF}, \mathrm{TP}$ \\
\hline Cerastium arvense $\mathrm{L}$. & 3 & $\mathrm{H}$ & Ap. & M & HF, TP, SI \\
\hline Cerastium holosteoides Fr. em. Hyl. & 2 & $\mathrm{~T} 2, \mathrm{H}$ & Ap. & M & HF, TP, SI \\
\hline Spergula arvensis L. & 1 & $\mathrm{~T} 1$ & Arch. & SG & TP, HF \\
\hline Spergularia rubra (L.) J. Pres et C. Presl. & 2 & T1-H & Arch. & SG & TP, HF \\
\hline $\begin{array}{l}\text { Melandrium album (Mill.) Garcke } \\
\text { (Silene alba (Mill.) E.H.L Krause) }\end{array}$ & 2 & $\mathrm{H}$ & Ap. & $\mathrm{RD}$ & RS, BS, HF, SI \\
\hline Ranunculaceae & & & & & \\
\hline Consolida regalis Gray & 1 & $\mathrm{~T} 1$ & Arch. & SG & TP, HF \\
\hline Myosurus minimus $\mathrm{L}$. & 3 & $\mathrm{~T} 1$ & Ap. & SW & $\mathrm{HF}$ \\
\hline Ranunculus repens $\mathrm{L}$. & 3 & $\mathrm{H}$ & Ap. & M & HF, BS, SI, D \\
\hline Papaveraceae & & & & & \\
\hline Papaver argemone $\mathrm{L}$. & 1 & $\mathrm{~T} 1$ & Arch. & SG & $\mathrm{TP}, \mathrm{HF}$ \\
\hline Papaver dubium $\mathrm{L}$. & 1 & T1 & Arch. & SG & TP, HF, RS \\
\hline Papaver rhoeas L. & 2 & $\mathrm{~T} 1$ & Arch. & SG & $\mathrm{TP}, \mathrm{HF}$ \\
\hline Chelidonium majus $\mathrm{L}$. & 2 & $\mathrm{H}$ & Ap. & $\mathrm{SE}$ & BS \\
\hline
\end{tabular}


Synanthropic orchard flora in West Mazovia.....

\begin{tabular}{|c|c|c|c|c|c|}
\hline $\begin{array}{l}\text { Fumariaceae } \\
\text { Fumaria officinalis } \mathrm{L} .\end{array}$ & 1 & T1 & Arch. & SG & $\mathrm{HF}, \mathrm{TP}$ \\
\hline Brassicaceae & & & & & \\
\hline Rorippa sylvestris (L.) Besser & 3 & $\mathrm{H}$ & Ap. & $\mathrm{RD}$ & $\mathrm{HF}, \mathrm{RS}, \mathrm{BS}$ \\
\hline Barbarea vulgaris $\mathrm{R} . \mathrm{Br}$. & 1 & T2-H & Ap. & $\mathrm{M}, \mathrm{RD}$ & RS, D \\
\hline Sisymbrium officinale (L.) Scop. & 1 & T1 & Arch. & $\mathrm{RD}$ & RS, HF \\
\hline Sisymbrium loeselii $\mathrm{L}$. & 1 & $\mathrm{~T} 1-\mathrm{T} 2$ & Ken. & RD & $\mathrm{RS}, \mathrm{HF}$ \\
\hline Descurainia sophia (L.) Webb ex Prantl & 1 & T1-T2 & Arch. & RD & RS, BS, HF \\
\hline Erysimum cheiranthoides $\mathrm{L}$. & 1 & T1-T2 & Ap. & SG & RS, BS, HF \\
\hline Arabidopsis thaliana (L.) Heynh. & 1 & $\mathrm{~T} 1$ & Arch. & SG & $\mathrm{HF}, \mathrm{RS}, \mathrm{BS}$ \\
\hline $\begin{array}{l}\text { Alliaria petiolata (M. Bieb.) Cavara et } \\
\text { Grande }\end{array}$ & 1 & $\mathrm{~T} 2$ & Arch. & SE & \\
\hline Sinapis arvensis $\mathrm{L}$. & 1 & T1 & Arch. & SG & $\mathrm{TP}, \mathrm{HF}$ \\
\hline Armoracia rusticana G., M. et Sch. & 2 & $\mathrm{H}$ & Arch. & $\mathrm{RD}$ & RS, BS, HF \\
\hline Erophila verna (L.) Chevall. & 4 & $\mathrm{~T} 1$ & Ap. & SS & \\
\hline Thlaspi arvense L. & 2 & $\mathrm{~T} 1$ & Arch. & SG & $\mathrm{TP}, \mathrm{HF}$ \\
\hline Teesdalea nudicaulis (L.) R. Br. & 2 & $\mathrm{~T} 1$ & Arch. & $\mathrm{SS}, \mathrm{CW}$ & \\
\hline Capsella bursa-pastoris (L.) Med. & 5 & T1 & Arch. & $\mathrm{SG}, \mathrm{RD}$ & HF, TP \\
\hline Raphanus raphanistrum $\mathrm{L}$. & 3 & $\mathrm{~T} 1$ & Arch. & SG & $\mathrm{TP}, \mathrm{HF}$ \\
\hline $\begin{array}{l}\text { Crassulaceae } \\
\text { Sedum acre L. }\end{array}$ & 1 & $\mathrm{H}$ & Ap. & SS & $\mathrm{RS}$ \\
\hline Rosaceae & & & & & \\
\hline Rosa canina $\mathrm{L}$. & 1 & $\mathrm{~N}$ & Ap. & SE & BS, D \\
\hline Rubus caesius $\mathrm{L}$. & 1 & Ch. & Ap. & DW, SE & BS, RS \\
\hline Potentilla anserina $\mathrm{L}$. & 3 & $\mathrm{H}$ & Ap. & M & HF, BS, SI \\
\hline Potentilla reptans $\mathrm{L}$. & 3 & $\mathrm{H}$ & Ap. & M & HF, BS, SI \\
\hline Geum urbanum $\mathrm{L}$. & 1 & $\mathrm{H}$ & Ap. & SE, DW & $\mathrm{BS}$ \\
\hline Fabaceae & & & & & \\
\hline Vicia angustifolia Scop. & 1 & $\mathrm{~T} 1$ & Arch. & M & $\mathrm{TP}, \mathrm{HF}$ \\
\hline Vicia hirsuta (L.) S.F. Gray & 2 & $\mathrm{~T} 1$ & Arch. & SG & TP, HF \\
\hline Vicia tetrasperma (L.) Schreb. & 1 & $\mathrm{~T} 1$ & Arch. & SG & TP, HF \\
\hline Vicia villosa Roth. & 3 & $\mathrm{~T} 1$ & Arch. & SG & $\mathrm{TP}, \mathrm{HF}, \mathrm{BS}$ \\
\hline Vicia cracca $\mathrm{L}$. & 2 & $\mathrm{G}$ & Ap. & M & $\mathrm{BS}, \mathrm{HF}$ \\
\hline Lathyrus pratensis $\mathrm{L}$. & 2 & $\mathrm{H}$ & Ap. & M & RS, SI \\
\hline Melilotus alba Medik. & 1 & $\mathrm{~T} 2$ & Ap. & RD & RS, BS, D \\
\hline Medicago lupulina $\mathrm{L}$. & 3 & $\mathrm{~T} 1-\mathrm{T} 2$ & Ap. & $\mathrm{XS}, \mathrm{M}$ & RS, SI \\
\hline Trifolium arvense $\mathrm{L}$. & 1 & $\mathrm{~T} 1-\mathrm{T} 2$ & Ap. & SS & SI, RS \\
\hline Trifolium dubium Sibth. & 1 & $\mathrm{~T} 1$ & Ap. & M & SI, RS \\
\hline Trifolium repens $\mathrm{L}$. & 4 & $\mathrm{H}$ & Ap. & M & SI, HF \\
\hline Trifolium hybridum $\mathrm{L}$. & 1 & $\mathrm{H}$ & Ap. & M & \\
\hline Trifolium pratense $\mathrm{L}$. & 2 & $\mathrm{H}$ & Ap. & M & SI, BS \\
\hline Geraniaceae & & & & & \\
\hline Geranium pusillum Burm. F. ex L. & 4 & T1 & Arch. & SG & $\mathrm{HF}, \mathrm{TP}, \mathrm{SI}$ \\
\hline Geranium molle L. & 1 & $\mathrm{~T} 1$ & Arch. & & \\
\hline Erodium cicutarium (L.) L'Hér. & 2 & $\mathrm{~T} 1$ & Ap. & XS, SG & RS, HF, TP, SI \\
\hline Sapindaceae & & & & & \\
\hline Acer negundo L. & 1 & M & Ken. & DW & $\mathrm{BS}, \mathrm{HF}$ \\
\hline Balsaminaceae & & & & & \\
\hline Impatiens parviflora $\mathrm{DC}$. & 1 & $\mathrm{~T} 1$ & Ken. & DW, SE & BS \\
\hline Euphorbiaceae & & & & & \\
\hline Euphorbia helioscopia L. & 2 & $\mathrm{~T} 1$ & Arch. & SG & $\mathrm{TP}, \mathrm{HF}$ \\
\hline Euphorbia peplus L. & 1 & $\mathrm{~T} 1$ & Arch. & SG & $\mathrm{TP}, \mathrm{HF}$ \\
\hline Malvaceae & & & & & \\
\hline Malva neglecta $\mathrm{L}$. & 3 & T2-H & Arch. & $\mathrm{RD}$ & $\mathrm{HF}, \mathrm{BS}$ \\
\hline Malva pusilla $\mathrm{Sm}$. & 1 & $\mathrm{~T} 1-2, \mathrm{H}$ & Arch. & RD & RS, BS, HF \\
\hline Malva sylvestris $\mathrm{L}$. & 1 & $\mathrm{~T} 2-\mathrm{H}$ & Arch. & $\mathrm{RD}$ & RS, BS, HF \\
\hline
\end{tabular}




\section{J. Lisek}

\begin{tabular}{|c|c|c|c|c|c|}
\hline $\begin{array}{l}\text { Clusiaceae (Hypericaceae) } \\
\text { Hypericum perforatum } \mathrm{L} \text {. }\end{array}$ & 2 & $\mathrm{H}$ & Ap. & DW & $\mathrm{BS}, \mathrm{HF}$ \\
\hline $\begin{array}{l}\text { Violaceae } \\
\text { Viola arvensis Murray } \\
\text { Viola tricolor L. }\end{array}$ & $\begin{array}{l}4 \\
1\end{array}$ & $\begin{array}{l}\text { T1 } \\
\text { T1 }\end{array}$ & $\begin{array}{l}\text { Arch. } \\
\text { Ap. }\end{array}$ & $\begin{array}{l}\text { SG } \\
\mathrm{N}\end{array}$ & $\begin{array}{l}\text { HF, TP } \\
\text { BS, HF, TP }\end{array}$ \\
\hline $\begin{array}{l}\text { Lythraceae } \\
\text { Lythrum salicilaria } \mathrm{L} .\end{array}$ & 1 & $\mathrm{H}$ & Ap. & $\mathrm{M}, \mathrm{SW}$ & BS, D \\
\hline $\begin{array}{l}\text { Onagraceae } \\
\text { Epilobium hirsutum } \text { L. } \\
\text { Epilobium adenocaulon Hausskn. } \\
\text { Epilobium adnatum } \text { Griseb. } \\
\text { Epilobium montanum L. } \\
\text { Epilobium roseum } \text { Schreb. } \\
\text { Chamaenerion angustifolium (L.) Scop. }\end{array}$ & $\begin{array}{l}1 \\
5 \\
2 \\
2 \\
1 \\
1\end{array}$ & $\begin{array}{l}\mathrm{H} \\
\mathrm{H} \\
\mathrm{H} \\
\mathrm{H} \\
\mathrm{H} \\
\mathrm{H}\end{array}$ & $\begin{array}{l}\text { Ap. } \\
\text { Ken. } \\
\text { Ap. } \\
\text { Ap. } \\
\text { Ap. } \\
\text { Ap. }\end{array}$ & $\begin{array}{l}\text { M, RD } \\
\text { RD, SW } \\
\text { M, SW } \\
\text { DW, SE } \\
\text { SW, SE } \\
\text { SE }\end{array}$ & $\begin{array}{l}\text { BS, D, HF } \\
\text { HF, BS } \\
\text { BS, D, HF } \\
\text { HF, BS } \\
\text { HF, BS, D } \\
\text { BS }\end{array}$ \\
\hline $\begin{array}{l}\text { Apiaceae } \\
\text { Aegopodium podagraria } \mathrm{L} . \\
\text { Aethusa cynapium } \mathrm{L} . \\
\text { Chaerophyllum temulum } \mathrm{L} \text {. } \\
\text { Heracleum sphondylium } \mathrm{L} . \\
\text { Daucus carota } \mathrm{L} \text {. }\end{array}$ & $\begin{array}{l}2 \\
1 \\
2 \\
2 \\
2\end{array}$ & $\begin{array}{l}\mathrm{H} \\
\mathrm{T} 1 \\
\mathrm{~T} 2 \\
\mathrm{~T} 2-\mathrm{H} \\
\mathrm{T} 2\end{array}$ & $\begin{array}{l}\text { Ap. } \\
\text { Arch. } \\
\text { Ap. } \\
\text { Ap. } \\
\text { Ap. }\end{array}$ & $\begin{array}{l}\text { DW } \\
\text { RD } \\
\text { DW } \\
\text { M } \\
\text { M }\end{array}$ & $\begin{array}{l}\text { BS, HF, SI } \\
\text { BS } \\
\text { BS } \\
\text { HF, BS } \\
\text { BS, HF }\end{array}$ \\
\hline $\begin{array}{l}\text { Primulaceae } \\
\text { Anagalis arvensis } \mathrm{L} \text {. }\end{array}$ & 1 & $\mathrm{~T} 1$ & Arch. & SG & $\mathrm{TP}, \mathrm{HF}$ \\
\hline $\begin{array}{l}\text { Rubiaceae } \\
\text { Galium aparine L. } \\
\text { Gallium album Mill. }\end{array}$ & $\begin{array}{l}4 \\
2\end{array}$ & $\begin{array}{l}\mathrm{T} 1 \\
\mathrm{H}\end{array}$ & $\begin{array}{l}\text { Ap. } \\
\text { Ap. }\end{array}$ & $\begin{array}{l}\text { SE } \\
M\end{array}$ & $\begin{array}{l}\text { HF, BS, TP } \\
\text { BS, D }\end{array}$ \\
\hline $\begin{array}{l}\text { Adoxaceae } \\
\text { Sambucus nigra L. }\end{array}$ & 1 & $\mathrm{~N}$ & Ap. & SE & BS \\
\hline $\begin{array}{l}\text { Convolvulaceae } \\
\text { Convolvulus arvensis } \mathrm{L} . \\
\text { Calystegia sepium (L.) R. Br. }\end{array}$ & $\begin{array}{l}4 \\
1\end{array}$ & $\begin{array}{l}\mathrm{G} \\
\mathrm{G}\end{array}$ & $\begin{array}{l}\text { Ap. } \\
\text { Ap. }\end{array}$ & $\begin{array}{l}\text { RD } \\
\text { SE }\end{array}$ & $\begin{array}{l}\mathrm{HF}, \mathrm{BS}, \mathrm{RS} \\
\mathrm{BS}\end{array}$ \\
\hline $\begin{array}{l}\text { Boraginaceae } \\
\text { Lithospermum arvense L. } \\
\text { Anchusa arvensis (L.) M. Bieb. } \\
\text { Echium vulgare L. } \\
\text { Myosostis stricta Link ex Roem. et Schult. } \\
\text { Myosostis arvensis (L.) Hill }\end{array}$ & $\begin{array}{l}1 \\
1 \\
1 \\
2 \\
3\end{array}$ & $\begin{array}{l}\mathrm{T} 1 \\
\mathrm{~T} 1 \\
\mathrm{~T} 2 \\
\mathrm{~T} 1 \\
\mathrm{~T} 1\end{array}$ & $\begin{array}{l}\text { Arch. } \\
\text { Arch. } \\
\text { Ap. } \\
\text { Arch. } \\
\text { Arch. }\end{array}$ & $\begin{array}{l}\text { SS, RD } \\
\text { SG, SS } \\
\text { RD } \\
\text { SG } \\
\text { SG }\end{array}$ & $\begin{array}{l}\text { RS, HF } \\
\text { RS, BS } \\
\text { RS } \\
\text { TP, HF, SI } \\
\text { HF, TP, D }\end{array}$ \\
\hline $\begin{array}{l}\text { Solanaceae } \\
\text { Solanum nigrum L. em. Mill. }\end{array}$ & 3 & $\mathrm{~T} 1$ & Arch. & SG & $\mathrm{HF}, \mathrm{TP}$ \\
\hline $\begin{array}{l}\text { Scrophulariaceae } \\
\text { Linaria vulgaris Mill. } \\
\text { Veronica chamaedrys L. } \\
\text { Veronica arvensis L. } \\
\text { Veronica persica } \text { Poir. } \\
\text { Veronica hederifolia } \mathrm{L} \text {. }\end{array}$ & $\begin{array}{l}1 \\
3 \\
3 \\
3 \\
1\end{array}$ & $\begin{array}{l}\mathrm{G} \\
\mathrm{H} \\
\mathrm{T} 1 \\
\mathrm{~T} 1 \\
\mathrm{~T} 1\end{array}$ & $\begin{array}{l}\text { Ap. } \\
\text { Ap. } \\
\text { Arch. } \\
\text { Ken. } \\
\text { Arch. }\end{array}$ & $\begin{array}{l}\text { SS } \\
\text { M } \\
\text { SG } \\
\text { SG } \\
\text { SE }\end{array}$ & $\begin{array}{l}\text { RS, BS } \\
\text { SI, BS, HF } \\
\text { TP, HF } \\
\text { HF, TP } \\
\text { BS, HF }\end{array}$ \\
\hline $\begin{array}{l}\text { Lamiaceae } \\
\text { Glechoma hederaceae } \mathrm{L} \text {. } \\
\text { Galeopsis tetrahit } \mathrm{L} . \\
\text { Lamium purpureum } \mathrm{L} . \\
\text { Lamium amplexicaule } \mathrm{L} \text {. } \\
\text { Stachys palustris } \mathrm{L} . \\
\text { Ballota nigra } \mathrm{L} . \\
\text { Mentha arvensis } \mathrm{L} .\end{array}$ & $\begin{array}{l}2 \\
2 \\
4 \\
3 \\
1 \\
1 \\
1\end{array}$ & $\begin{array}{l}\mathrm{H} \\
\text { T1 } \\
\text { T1 } \\
\text { T1 } \\
\text { G } \\
\text { Ch, H } \\
\text { G }\end{array}$ & $\begin{array}{l}\text { Ap. } \\
\text { Ap. } \\
\text { Arch. } \\
\text { Arch. } \\
\text { Ap. } \\
\text { Arch. } \\
\text { Ap. }\end{array}$ & $\begin{array}{l}\text { SE } \\
\text { DW,CW } \\
\text { SG } \\
\text { SG } \\
\text { SW } \\
\text { RD } \\
\text { M, SW }\end{array}$ & $\begin{array}{l}\text { BS, HF, SI } \\
\text { TP, HF, BS } \\
\text { HF, TP } \\
\text { TP, HF } \\
\text { BS, D, HF } \\
\text { BS, RS } \\
\text { BS, D, HF }\end{array}$ \\
\hline
\end{tabular}




\begin{tabular}{|c|c|c|c|c|c|}
\hline Plantaginaceae & & & & & \\
\hline Plantago major $\mathrm{L}$. & 4 & $\mathrm{H}$ & Ap. & $\mathrm{RD}$ & SI, RS, TR, HF \\
\hline Plantago lanceolata $\mathrm{L}$. & 4 & $\mathrm{H}$ & Ap. & M & SI, HF \\
\hline Asteraceae & & & & & \\
\hline Solidago canadensis $\mathrm{L}$. & 3 & $\mathrm{G}, \mathrm{H}$ & Ken. & $\mathrm{RD}$ & $\mathrm{BS}, \mathrm{HF}$ \\
\hline Solidago gigantea Aiton & 2 & G & Ken. & RD & $\mathrm{BS}, \mathrm{HF}$ \\
\hline Conyza canadensis (L.) Cronq. & 5 & $\mathrm{~T} 1-\mathrm{T} 2$ & Ken. & RD & $\mathrm{HF}, \mathrm{TP}, \mathrm{RS}$ \\
\hline Erigeron annuus (L.) Pers. & 1 & $\mathrm{~T} 2-\mathrm{H}$ & Ken. & $\mathrm{RD}$ & BS, HF \\
\hline Gnaphalium uliginosum $\mathrm{L}$. & 1 & $\mathrm{~T} 1$ & Ap. & M, SW & TP, HF, D, SI \\
\hline Bidens tripartita L. & 1 & T1 & Ap. & M, SW & $\mathrm{BS}, \mathrm{HF}$ \\
\hline Galinsoga parviflora Cav. & 3 & T1 & Ken. & SG & $\mathrm{TP}, \mathrm{HF}$ \\
\hline Galinsoga ciliata (Raf.) S.F. Blake & 2 & $\mathrm{~T} 1$ & Ken. & SG & TP, HF \\
\hline Anthemis arvensis $\mathrm{L}$. & 2 & T1 & Arch. & SG & $\mathrm{TP}, \mathrm{HF}$ \\
\hline Achillea millefolium $\mathrm{L}$. & 3 & & Ap. & M & SI, RS, HF \\
\hline $\begin{array}{l}\text { Matricaria maritima L. ssp. inodora } \\
\text { (L.) (Matricaria perforata Mérat) }\end{array}$ & 3 & T1-2, H & Arch. & SG & HF, TP, BS, RS \\
\hline $\begin{array}{l}\text { Chamomilla suaveolens (Pursh) Rydb. } \\
\text { (Matricaria discoidea DC.) }\end{array}$ & 4 & $\mathrm{~T} 1$ & Ken. & $\mathrm{RD}$ & RS, TR, HF \\
\hline Chamomilla recutita $\mathrm{L}$. Rauschert & 2 & T1 & Arch. & SG & RS, TP, HF \\
\hline Tanacetum vulgare $\mathrm{L}$. & 2 & $\mathrm{H}$ & Ap. & $\mathrm{RD}$ & RS, BS \\
\hline Artemisia vulgaris L. & 3 & $\mathrm{H}$ & Ap. & $\mathrm{RD}$ & $\mathrm{BS}, \mathrm{HF}$ \\
\hline Tusillago farfara $\mathrm{L}$. & 1 & $\mathrm{G}$ & Ap. & $\mathrm{RD}$ & RS, BS \\
\hline Senecio vulgaris $\mathrm{L}$. & 4 & $\mathrm{~T} 1$ & Arch. & SG & $\mathrm{HF}, \mathrm{TP}$ \\
\hline Senecio jacobaea $\mathrm{L}$. & 2 & $\mathrm{~T} 1$ & Ap. & XS & RS, HF, BS \\
\hline Arctium tomentosum Mill. & 2 & $\mathrm{~T} 2$ & Ap. & $\mathrm{RD}$ & $\mathrm{RS}, \mathrm{BS}$ \\
\hline Cirsium vulgare (Savi) Ten. & 1 & $\mathrm{~T} 2$ & Ap. & RD & BS, SI, HF \\
\hline Cirsium arvense (L.) Scop. & 3 & $\mathrm{G}$ & Ap. & RD & $\mathrm{HF}, \mathrm{TP}, \mathrm{BS}$ \\
\hline Centaurea jacea $\mathrm{L}$. & 1 & $\mathrm{H}$ & Ap. & M & RS \\
\hline Cichorium intybus $\mathrm{L}$. & 2 & $\mathrm{H}$ & Arch. & RD & RS \\
\hline Hypochoeris radicata $\mathrm{L}$. & 3 & $\mathrm{H}$ & Ap. & $\mathrm{SS}, \mathrm{CW}$ & SI, RS \\
\hline Hypochoeris glabra L. & 1 & $\mathrm{~T} 1$ & Arch. & SS & SI, RS \\
\hline Tragopogon pratensis $\mathrm{L}$. & 1 & $\mathrm{~T} 2$ & Ap. & M & SI, RS \\
\hline Leontodon autumnalis $\mathrm{L}$. & 3 & $\mathrm{H}$ & Ap. & $\mathrm{M}, \mathrm{RD}$ & SI, HF \\
\hline Leontodon hispidus L. & 2 & $\mathrm{H}$ & Ap. & M & \\
\hline Picris hieracioides L. & 1 & $\mathrm{H}$ & Ap. & $\mathrm{RD}$ & SI, RS \\
\hline Sonchus oleraceus L. & 3 & $\mathrm{~T} 1$ & Arch. & SG & TP, HF, BS \\
\hline Sonchus asper (L.) Hill & 2 & $\mathrm{~T} 1$ & Arch. & SG & BS, HF, TP \\
\hline Sonchus arvensis L. & 2 & $\mathrm{H}$ & Ap. & SG & HF, BS, TP \\
\hline Lactuca serriola $\mathrm{L}$. & 3 & $\mathrm{~T} 2$ & Arch. & $\mathrm{RD}$ & HF, RS, BS \\
\hline Taraxacum officinale $\mathrm{F}$. H. Wigg. & 5 & $\mathrm{H}$ & Ap. & M & HF, SI \\
\hline Lapsana communis L. & 1 & $\mathrm{~T} 1$ & Ap. & SE & BS, HF, SI \\
\hline Crepis biennis L. & 3 & $\mathrm{~T} 2$ & Ap. & $\mathrm{M}$ & SI, HF \\
\hline Crepis tectorum $\mathrm{L}$. & 3 & T1-2 & Ap. & SS & SI, RS \\
\hline Crepis capillaris (L.) Wallr. & 3 & $\mathrm{~T} 1-2$ & Ap. & & SI, RS \\
\hline Hieracium pilosella $\mathrm{L}$. & 3 & $\mathrm{H}$ & Arch. & SS, XS & SI, RS \\
\hline Juncaceae & & & & & \\
\hline Juncus conglomeratus $\mathrm{L}$. & 1 & $\mathrm{H}$ & Arch. & M, SW & $\mathrm{D}, \mathrm{SI}$ \\
\hline Cyperaceae & & & & & \\
\hline Carex hirta $\mathrm{L}$. & 1 & G & Ap. & $\mathrm{SW}, \mathrm{RD}$ & $\mathrm{D}, \mathrm{SI}$ \\
\hline Poaceae & & & & & \\
\hline Festuca pratensis Huds. & 3 & $\mathrm{H}$ & Ap. & M & SI, BS \\
\hline Festuca rubra $\mathrm{L}$. & 4 & $\mathrm{H}$ & Ap. & M & SI, BS \\
\hline Lolium perenne $\mathrm{L}$. & 5 & $\mathrm{H}$ & Ap. & $\mathrm{M}, \mathrm{RD}$ & SI, HF, BS \\
\hline Lolium multiflorum Lam. & 2 & $\mathrm{H}$ & Ken. & $\mathrm{RD}$ & SI, BS \\
\hline Poa compressa $\mathrm{L}$. & 2 & $\mathrm{H}$ & Ap. & SS, XS & SI, BS \\
\hline Poа аппиа $\mathrm{L}$. & 5 & $\mathrm{~T} 1$ & Ap. & $\mathrm{M}, \mathrm{RD}$ & HF, TP, RS, TR \\
\hline Poa pratensis $\mathrm{L}$. & 4 & $\mathrm{H}$ & Ap. & M & SI, BS \\
\hline Poa angustifolia $\mathrm{L}$. & 3 & $\mathrm{H}$ & Ap. & M & SI, BS \\
\hline Poa trivialis $\mathrm{L}$. & 2 & $\mathrm{H}$ & Ap. & M & SI, BS \\
\hline Dactylis glomerata $\mathrm{L}$. & 3 & $\mathrm{H}$ & Ap. & M & SI, BS \\
\hline Apera spica-venti (L.) P. Beauv. & 2 & T1 & Arch. & SG & $\mathrm{TP}$ \\
\hline Bromus sterilis L. & 2 & $\mathrm{~T} 2$ & Arch. & SE & $\mathrm{HF}, \mathrm{BS}$ \\
\hline
\end{tabular}




\begin{tabular}{|l|l|l|l|l|l|}
\hline Bromus inermis Leyss. & 3 & G & Ap. & RD & HF, BS \\
Bromus hordeaceus L. & 4 & T2 & Ap. & RD & HF, BS \\
Agropyron repens L. & 4 & G & Ap. & RD & HF, BS, TP \\
Hordeum murinum L. & 3 & T2 & Arch. & RD & RS, BS \\
Holcus mollis L. & 2 & G & Ap. & SS & SI, BS \\
Agrostis capillaris L. & 3 & H & Ap. & SS, XS & SI, HF, BS \\
Agrostis gigantea Roth & 2 & G & Ap. & M & HF, BS \\
Calamagrostis epigejos (L.) Roth & 1 & G & Ap. & DW, CW & D, BS \\
Phleum pretense L. & 2 & H & Ap. & M & SI, BS \\
Alopecurus pratensis L. & 3 & H & Ap. & M & SI, BS \\
Alopecurus aequalis Sobol. & 1 & T1 & Ap. & SW & SI, BS \\
Echinochloa crus galli (L.) P. Beauv. & 4 & T1 & Arch. & SG & HF, TP \\
Digitaria sanguinalis (L.) Scop. & 3 & T1 & Arch. & SG & HF, TP \\
Setaria pumila (Poir.) Roem et Schult. & 3 & T1 & Arch. & SG & HF, TP \\
Setaria viridis (L.) P. Beauv. & 2 & T1 & Arch. & SG & HF, TP \\
\hline
\end{tabular}

\section{Explanation}

Frequency of occurrence in the investigated area:

1 - occasionally; 2 - rarely; 3 - average; 4 - often; 5 - very often (> 50.1\% of sites).

Life form according to Raunkiaer:

- perennials: $\mathrm{M}$ - megaphanerophyte - tree; $\mathrm{N}$ - nanophanerophyte - shrub; $\mathrm{Ch}-$ herbaceous chamaephyte; $\mathrm{G}$ - geophyte; $\mathrm{H}$ - hemicryptophyte;

- short-lived: T1 - annual therophyte; T2 - biennial therophyte.

Geographical-historical classification:

Ap. - apophyte; Arch. - archeophyte; Ken. - kenophyte; Erg. - ergasiophyte.

Socio-ecological groups:

DW - deciduous woodland species; CD - coniferous woodland species; SE - shrub edges species; M - meadow species; SS - sandy sward species; XS - xerothermic sward species; RD - ruderal species; SG - segetal species; SW - swamp species; N species of under classification.

Sites of occurrence in investigated orchards:

TP - tillage places; HF - herbicide fallow under trees; SI - swarm of inter-rows; TR trodden and rutty places; RS - roadsides: BS - boundary stripes; D - drainage ditches.

examined orchards belonged to 39 botanical families. Most numerously represented were: Asteraceae - 39 species (21\%), Poaceae - 27 species (15\%), Brassicacea - 15 species $(8 \%)$, Fabaceae - 13 species (7\%).

Participation of Raunkiaer's life forms. Ninety-three species $(50 \%$ of the total number of plants collected) were therophytes $(T)$, i.e. short-lived forms, mostly annuals. Fifty-eight species $(31 \%)$ were classified as hemicryptophytes $(\mathrm{H})$, and 19 species $(10 \%)$ as geophytes (G). Nine species (5\%), among others, Malva sp., Heracleum sphondylium, Erigeron annuus, Matricaria maritima constituted both short lived forms and long lived forms. The rest of the longlived species (perennials) were classified as megaphanerophyte - 2 species; nanophanerophyte -2 ; chamaephyte/hemicryptophyte -1 ; geophyte/hemicryptophyte -1 . 
Geographical-historical classification. Flora of the evaluated orchards consisted mostly of apophytes (native plants), of which 110 species were collected $(59 \%)$. There was also the noted occurrence of 61 species of archeophytes (33\% of all species), 14 kenophytes (8\%) and 1 ergasiophyte (Juglans regia). From the 14 kenophytes, 9 originated in America, 3 (Sisymbrium loeselii, Veronica persica and Impatiens parviflora) from Asia, 1 (Chamomilla suaveolens) in the north-western part of America and East Asia and 1 (Lolium multiflorum) in the Mediterranean region.

Socio-ecological classification. The predominant species in the flora of the examined orchards were typically segetal, and there were 49 (26\%) of these segetal species. There was also a substantial share of typically ruderal species - $39(21 \%)$, meadow species - 35 (19\%), sandy and xerothermic sward species - $13(7 \%)$ as well as shrub edges species -10 $(5 \%)$. Species typical of other socioecological groups were not numerous.

Preferences of site. Weed occurrence at the particular sites of the orchard was presented in Table 1 . Number of species in the sites assessed was as following: herbicide fallow - 128, boundary stripes -100 , tillage places 61, swarm of inter-rows - 58, roadsides -52 , drainage ditches -17 . Trodden and rutty places belonged to sites occupied by the most limited number of species -4 . Twenty species occurred on only one site, e.g. Sedum acre in roadsides. Other species occurred on at least two sites.

\section{DISCUSSION}

The number of species of synanthropic plants occurring in the orchards of Central Poland did not diverge substantially either from the number noted in the orchards near Szczecin (in the north-western part of Poland - 162 species) (Wróbel, 1999), or from the number noted in the orchards of the Nile Delta in Egypt - 169 species (Mashaly and Awad, 2003). Especially important from the practical point of view, is the occurrence of plants of large biomass under branches of trees: in the herbicide fallow stripes, as well as in the swarms of inter-rows, which are considered weeds in the growing season. The same weeds should be regarded as most significant both in the commercial, conventional orchards of the central part of Poland and in the orchards treated with herbicides of the north-western (Wróbel, 1999) and eastern (region of Lublin) parts of Poland (Lipecki, 2004). In this group, among others, are: Equisetum arvense, Chenopodium album, Amaranthus retroflexus, Stellaria media, Capsella bursapastoris, Convolvulus arvensis, Epilobium adenocaulon, Taraxacum officinale, Conyza canadensis, Poa annua, and Echinochloa crus-galli. In the flora of young organic orchards in Skierniewice (central part of Poland), those weeds which consist of 25 species, such as Stellaria media, Capsella bursa-pastoris, and Geranium pusillum, and are typical of 
the regional orchards where herbicides are applied, did not occur (Mika, 2004). In the orchards with the IPM, located in the Warmia and Mazury Province (in the north-eastern part of Poland), predominant were Senecio vulgaris and Stellaria media, whereas Chenopodium album, Urtica urens, Vicia tetrasperma, Capsella bursa-pastoris, Equisetum arvense, Echinochloa crus-galli, Polygonum lapatifolium, Epilobium adenocaulon, Poa annua and Taraxacum officinale were less numerous (Markuszewski and Kopytowski, 2008).

Some species like Convolvulus arvensis are cosmopolitan plants and occur as weeds in the orchards of various countries: Argentina (Conticello and Gandullo, 1991); Egypt (Mashaly and Awad, 2003), Bulgaria (Tasseva, 2005), and Turkey (Ustuner and Ustuner, 2011). Chenopodium album is an example of a cosmopolitan oneyear weed, frequently occurring in the orchards of South Korea (Jung et al., 1997), Argentina (Novo et al., 2000), New Zealand (Harrington et al., 2002), Canada (Rifai et al., 2002), Egypt (Mashaly and Awad, 2003), Bulgaria (Tasseva, 2005), and Turkey (Ustuner and Ustuner, 2011). To the group of species commonly occurring in the orchards of different countries and continents belong, according to the previously cited authors: Stellaria media, Capsella bursa-pastoris, Echinochloa crusgalli, and Polygonum aviculare. This means that the anthropogenic factor - the technology of cultivating the fruit trees - has an important influence on the species composition of orchard synanthropic flora, including weeds. The success of particular species, measured by the number of plants, depends on tolerance to herbicides, long period of germination (Geranium pusillum, Viola arvensis, Capsella bursapastoris), large number of seeds (Chenopodium album), anemochores (Asteraceae, Epilobium adenocaulon), and permanence of seeds in the soil (Amaranthus retroflexus, Echinochloa crus-galli (Tymrakiewicz, 1976; Mowszowicz, 1984). The common occurrence of perennials, such as Equisetum arvense, Epilobium adenocaulon is connected to the low sensitivity of the plants of these species to herbicides applied in orchards (Lisek, 2001). Orchards with a varied technology of soil management and varied lighting of the inter-rows and under the branches of trees, create conditions proper for the development of plants belonging to the different socioecological group. Plant mowing in the boundary stripes stimulated the development of flora characteristic of meadows and swarms, and their afforestation supported the development of species typical of shrub edges and deciduous woodland. The composition of flora in the examined orchards was modified by environmental conditions, succession, and the age of the orchard. A lack of tillage over the span of many years, favoured the development of perennial weeds; their compensation. Simultaneous occurrence of therophytes, hemicryptophytes, and geophytes creates difficulties for 
providing a practical way to limit the number of weeds. The preferred method of protection integrates the various methods of weed control.

\section{CONCLUSIONS}

1. Synanthropic flora of orchards of central Poland near Skierniewice, Łowicz and Grójec consisted of 186 species of vascular plants belonging to 39 botanical families. Most frequently represented were Asteraceae (21\%), Poaceae (15\%), Brassicacea (8\%) and Fabaceae (7\%).

2. Around $60 \%$ of the species found in the examined orchards occurred occasionally or rarely, whereas $15 \%$ of the species were noted frequently or very frequently. Frequently occurring species were numerously represented in the herbicide fallow field under the trees, so they should be acknowledged as the most significant orchard weeds.

3. The most numerous group of the analysed flora constituted of therophytes $(50 \%)$, which predominated over hemicryptophytes (31\%) and geophytes (10\%).

4. Apophytes - native species $(59 \%)$ predominated over archeophytes (33\%) and kenophytes (8\%).

5. Among the vascular flora of the orchards examined, segetal species (26\%) predominated over ruderal (21\%) and meadow (19\%) species.

\section{REFERENCES}

Braun-Blanquet J. 1964. Pflanzensoziologie. $3^{\text {rd }}$ ed. Soringerverlag, Wien, p. 865.
Conticello L., Gandullo R. 1991. Survey of summer weeds in the upper valley of Rio Negro y Neuqen. ASSOC. ARGENTINA PARA EL CONTROL DE MALEZAS 1: 19-26.

Ellenberg H., Weber H., Düll R., Wirth V., Werner W., Paulissen D. 1992. Zeigerwerte von Pflanzen in Mitteleuropa. Scr. Geobot. 18, Göttingen, p. 258.

Harrington K.C., Hartley M.J., Rahman A., James T.K. 2002. Strategies for controlling weeds in New Zealand apple orchard. Plant Prot. Soc. of Western Australia Inc., pp. 208-211.

Jung J.S., Lee J.S., Choi C. D. 1997. Weed occurrence in apple orchards in Korea. KOREAN J. WEED SCI., 17(2): 147-156.

Kornaś J. 1981. Oddziaływanie człowieka na florę: mechanizmy i konsekwencje. WIAD. BOT. 25(3): 165-182.

Lipecki J. 2004. Weeds in orchards in Lublin region (almost) twenty years later - preliminary report. J. FRUIT ORNAM. PLANT RES. 12: 105-111.

Lisek J. 2001. Podstawowe aspekty zwalczania chwastów w sadach na przełomie wieków. PROG. PLANT PROTEC./POST. OCHR. ROŚ. 41(1): 196-202.

Markuszewski B., Kopytowski J. 2008. Zachwaszczenie i koszty jego regulowania $\mathrm{w}$ sadzie jabłoniowym $\mathrm{z}$ produkcją integrowaną. ZESZ. NAUK. INST. SADOW. KWIAC. 16: 35-50.

Mashaly I.A., Awad E.R. 2003. Weed flora of orchards in the Nile delta, Egypt: floristic features. ASIAN J. PLANT SCI. 2(3): 314-324.

Mika A. 2004. The importance of biodiversity in natural environment and in fruit plantations. J. FRUIT ORNAM. PLANT RES. 12: 11-21. 
Mirek Z., Piękoś-Mirkowa H., Zając A., Zając M. 2002. Flowering plants and pteridophytes of Poland. A checklist. Krytyczna lista roślin naczyniowych Polski. Wyd. Bot. PAN, Kraków, p. 442.

Mowszowicz J. 1984. Krajowe chwasty polne i ogrodowe. PWRiL, Warszawa, p. 672 .

Novo R.J., Nobile R.A., Zaninetti M.R. 2000. Weeds in peach tree crops in Cordoba. HORT. ARGENTINA 18(44/45): 43-50.

Rifai M.N., Astatkie T., LackoBartosova M., Gadus J. 2002. Effect of two different thermal units and three types of mulch on weeds in apple orchards. J. ENVIRON. ENG. SCI. 1: 1-16.
Rutkowski L. 2007. Klucz do oznaczania roślin naczyniowych Polski niżowej. PWN, Warszawa, p. 814.

Tasseva V. 2005. Species composition of weed vegetation in different apple growing technologies. NOT. BOT. HORT. AGROBOT. CLUJ. 25: 59-64.

Tymrakiewicz W. 1976. Atlas chwastów. PWRiL, Warszawa, p. 439.

Ustuner T., Ustuner M. 2011. Investigation on different mulch materials and chemical control for controlling weds in apple orchard in Turkey. SCI. RES. ESS. 6(19): 3979-3985.

Wróbel M. 1999. Orchard flora in Szczecin surroundings. J. FRUIT ORNAM. PLANT RES. 7(3): 133-145.

Zając M., Zając A. 1992. A tentative list of segetal and ruderal apohytes in Poland. ZESZ. NAUK. UJ, KRAKÓW, 1059. PRACE BOT. 24: 7-23. 


\title{
FLORA SYNATROPIJNA SADÓW CENTRALNEJ POLSKI
}

\author{
Jerzy Lisek
}

\author{
S T R E S Z C Z E N I E
}

W sadach w centralnej Polsce w okolicy Skierniewic, Łowicza i Grójca badano florę synantropijną. W latach 2007-2010 wykonano 390 zdjęć fitosocjologicznych obejmujących ugór herbicydowy pod koronami drzew, murawę międzyrzędzi, ugór mechaniczny, uwrocia i miejsca wydeptywane, przydroża (pobocza dróg wewnętrznych), przypłocia i rowy melioracyjne $\mathrm{W}$ badanych sadach zarejestrowano występowanie 186 gatunków, należących do 39 rodzin botanicznych, z których najliczniejsze były Asteraceae (21\%), Poaceae (15\%), Brassicaceae (8\%) i Fabaceae (7\%). Spośród gatunków znalezionych w badanych sadach 60\% występowało okazjonalnie lub rzadko. 15\% gatunków, między innymi Equisetum arvense, Polygonum aviculare, Chenopodium album, Amaranthus retroflexus, Stellaria media, Capsella bursa-pastoris, Convolvulus arvensis, Viola arvensis, Epilobium adenocaulon, Conyza canadensis, Taraxacum officinale, Poa annua, Echinochloa crus-galli, występowało często lub bardzo często, przede wszystkim w ugorze herbicydowym i uznano je za najważniejsze chwasty sadów. Najliczniejszą grupę analizowanej flory stanowiły terofity $(50 \%)$, które przeważały nad hemikryptofitami (31\%) i geofitami (10\%). Apofity - gatunki rodzime (59\%) przeważały nad archeofitami (33\%) i kenofitami (8\%). Wśród flory naczyniowej badanych sadów przeważały gatunki segetalne (26\%), ruderalne $(21 \%)$ i łąkowe $(19 \%)$.

Słowa kluczowe: rośliny synantropijne, chwasty, sad 